\title{
Aspect grammatical et temps interne
}

\author{
Jacques Bres ${ }^{1 *}$ \\ ${ }^{1}$ Université Paul-Valéry, Montpellier 3, Praxiling, UMR 5267, Route de Mende, 34199, \\ Montpellier, Cedex 5
}

\begin{abstract}
Résumé. G. Guillaume définit l'aspect grammatical à partir de la notion de temps impliqué (1964[1933]) Nous lui empruntons cette notion, que nous renommons pour plus de lisibilité temps interne, et décrivons rapidement, à partir d'elle, le système aspectuel des temps verbaux de l'indicatif en français. Nous montrons dans un second temps que la notion de temps interne permet de rendre compte de façon consistante de la morphosyntaxe du participe passé, ainsi que de l'emploi narratif du passé composé.
\end{abstract}

\begin{abstract}
Guillaume defines grammatical aspect from the notion of 'temps impliqué' (involved time) (1964[1933]). While borrowing this notion, here renamed 'internal time' for more clarity, this paper gives a brief description of the aspectual system of French tenses in the indicative mood. The notion of 'internal time' will prove enlightening to account for the morpho-syntax of the past participle, as well as the narrative use of the passé compose.
\end{abstract}

Les recherches que nous développons sur les temps verbaux de l'indicatif depuis maintenant bientôt 30 ans ont pris principalement appui sur l'approche psychomécanique (Guillaume 1970 [1929], 1964 [1933]) - en réévaluation critique (Bres 1997a), et complémentairement sur l'approche praxématique (Lafont 1967, 1978), à partir desquelles nous avons interagi avec (et discuté) les différentes théorisations contemporaines. Nous aimerions dans cette communication faire travailler plus précisément et plus spécifiquement que nous ne l'avons fait un élément qui, dans nos travaux les plus récents, nous est apparu particulièrement heuristique : la notion guillaumienne de temps impliqué (Guillaume 1964 [1933]) (section 1.), dans la mesure où elle permet de décrire de façon pertinente la morpho-syntaxe du participe passé (Bres et Le Bellec 2017, 2019) (section 2.), ainsi que l'emploi narratif du passé composé (Bres 2020, sous presse) (section 3.).

\section{Temps interne, aspect, aspects}

Dans son ouvrage séminal, Temps et verbe, Guillaume avance que « le propre du verbe est d'être sous-tendu de temps » (1970 [1929] :7), «temps, qu'il contient intérieurement » (op. cit. : 15). Il développera plus précisément cette analyse dans l'article de 1964 [1933] à travers la distinction temps expliqué / temps impliqué :

\footnotetext{
*jacques.bres@univ-montp3.fr
} 
Le verbe est un sémantème qui implique et explique le temps. Le temps impliqué est celui que le verbe emporte avec soi, qui lui est inhérent, fait partie intégrante de sa substance et dont la notion est indissolublement liée à celle de verbe. Il suffit de prononcer le nom d'un verbe comme marcher pour que s'éveille dans l'esprit, avec l'idée d'un procès, celle du temps destiné à en porter la réalisation.

Le temps expliqué est autre chose. Ce n'est pas le temps que le verbe retient en soi par définition, mais le temps divisible en moments distincts - passé, présent, futur, et leurs interprétations, que le discours lui attribue. Cette distinction du temps impliqué et du temps expliqué coïncide exactement avec la distinction de l'aspect et du temps. (Guillaume 1964[1933] : 47-48).

Nous retenons cette distinction, que nous renommons par celle, plus lisible, de temps interne, qui concerne la catégorie de l'aspect, et de temps externe, qui concerne la catégorie du temps.

Reprenons, pour commencer, la représentation, partagée par différents chercheurs (i.a. Dik 1989, Tournadre 2004, Gosselin 2011), selon laquelle un procès peut être saisi selon trois phases, pré-processuelle, processuelle, et post-processuelle :

$$
\text { Phases pré-processuelle processuelle } \mathbf{E}_{\mathbf{t}} \text { post--------- }
$$

Figure 1. Les phases du procès

On a coutume de dire que l'intervalle $E_{\mathbf{i}}-E_{t}$ représente l'intervalle du procès. Précisons de la sorte : l'intervalle temporel du procès entre le début de sa réalisation $\mathrm{E}_{\mathbf{i}}$ et sa fin Et. À la différence de noms comme livre, roue ou viole, tout procès, en tant que représentation d'un événement ou d'un état, implique du temps : le temps interne de réalisation de la tension de la phase processuelle (définie comme «l'impression de mobilité progressive qui en est inséparable » (Guillaume 1970 [1929] : 15) depuis la tension totale en $E_{i}$ jusqu'à la détension en $E_{t}{ }^{i}$. Les différentes façons de saisir le temps interne du procès constituent les différents aspects d'une langue ${ }^{\mathrm{ii}}$.

Présentons rapidement les différentes façons de représenter le temps interne du procès en français. Tel que nous venons de le définir, il concerne la phase processuelle, et est signifié (principalement) par les formes simples ou synthétiques. Il s'agit de l'aspect tensif. Il peut également être saisi après son déroulement, dans la phase post-processuelle : à cela servent les formes composées ou analytiques. Il s'agit de l'aspect extensif, qui se prolonge de l'aspect bi-extensif. Ajoutons que, à l'aspect tensif, le temps interne peut être saisi globalement (de $\mathrm{E}_{\mathbf{i}}$ à $\mathrm{E}_{\mathbf{t}}$ ), cursivement (en un point de l'intervalle $\mathrm{E}_{\mathbf{i}}-\mathrm{E}_{\mathbf{t}}$ ), ou de façon non précisée, dite neutre. On distingue également, aux aspects extensif et bi-extensif, les saisies globale (à partir de $\mathrm{E}_{\mathrm{t}}$ ), cursive (au-delà de $\mathrm{E}_{\mathrm{t}}$ ), et neutre. Soit, pour le procès neiger au mode indicatif :

\begin{tabular}{|c|c|c|c|}
\hline & Aspect tensif & Aspect extensif & Aspect bi-extensif \\
\hline Aspect global & il neigea & il eut neigé & il eut eu neigé \\
\hline Aspect cursif & il neigeait & il avait neigé & il avait eu neigé \\
\hline Aspect neutre & $\begin{array}{l}\text { il neige, il neigera, } \\
\text { il neigerait }\end{array}$ & $\begin{array}{c}\text { il a / aura / aurait } \\
\text { neigé }\end{array}$ & $\begin{array}{c}\text { il a/aura/ aurait eu } \\
\text { neigé }\end{array}$ \\
\hline
\end{tabular}

Tableau 1. Formes synthétiques et analytiques à l'indicatif 
Par ailleurs, au cours de son histoire, la langue a développé, par grammaticalisation du verbe de déplacement aller, une façon de saisir le temps interne avant le début $\mathrm{E}_{\mathrm{i}}$ de son déroulement, à savoir dans la phase pré-processuelle : il s'agit de l'aspect prospectif: aller + infinitiffii. La langue a également développé une autre façon de saisir le temps interne après son déroulement, dans la phase post-processuelle : il s'agit de l'aspect rétrospectif: venir de + inf. ${ }^{\text {iv }}$. Aspects prospectif et rétrospectif peuvent représenter le temps du procès lui-même en tension (infinitif synthétique : il va neiger, il vient de neiger), comme (plus rarement) en extension (infinitif analytique : il va avoir neigé ; il vient d'avoir neigé) (Bres 2020). Et plus rarement encore, pour le prospectif, bi-extensivement.

\begin{tabular}{|c|c|c|c|}
\hline & Aspect tensif & Aspect extensif & Aspect bi-extensif $^{v}$ \\
\hline Aspect global & $\varnothing$ & $\varnothing$ & $\varnothing$ \\
\hline Aspect cursif & il allait neiger & il allait avoir neigé & $\begin{array}{c}\text { il allait avoir eu } \\
\text { neigé }\end{array}$ \\
\hline Aspect neutre & il va neiger & il va avoir neigé & il va avoir eu neigé \\
\hline
\end{tabular}

Tableau 2. Formes périphrastiques de l'aspect prospectif

\begin{tabular}{|c|c|c|}
\hline & Aspect tensif & Aspect extensif \\
\hline Aspect global & $\varnothing$ & $\varnothing$ \\
\hline Aspect cursif & il venait de neiger & $\begin{array}{c}\text { il venait d'avoir } \\
\text { neigé }\end{array}$ \\
\hline Aspect neutre & $\begin{array}{l}\text { il vient de neiger, } \\
\text { il viendra(it) de } \\
\text { neiger }\end{array}$ & $\begin{array}{c}\text { il vient d'avoir neigé } \\
\text { il viendra(it) d'avoir } \\
\text { neigé }\end{array}$ \\
\hline
\end{tabular}

Tableau 3. Formes périphrastiques de l'aspect rétrospectif

Ajoutons que cette répartition des aspects selon les phases du procès n'est pas figée : les formes de l'aspect prospectif, il va neiger et il allait neiger, qui initialement relevaient de la phase pré-processuelle, sont, au fil des siècles, venues concurrencer les formes tensives correspondantes de la phase processuelle : il neigera, il neigerait. On parle p. ex. dans les grammaires de il va neiger comme d'un futur proche. La forme post-processuelle il a neigé est venue concurrencer la forme tensive il neigea de la phase processuelle ${ }^{\mathrm{vi}}$.

On pourrait nous faire remarquer que, in fine, notre description des différents aspects à partir de la notion de temps interne n'est guère éloignée des descriptions grammaticales traditionnelles, ou du système particulièrement développé de L. Gosselin (1996, 2017), qui ne retiennent pas cette catégorie: p. ex., concernant le passé simple, il est traditionnellement dit qu'il représente le procès globalement, là où nous disons qu'il représente le temps interne $d u$ procès globalement. Nous-mêmes d'ailleurs, par souci de simplification, avons usé de la première formulation récemment dans notre essai de description du système des temps de l'indicatif (Azzopardi et Bres 2017).

Le temps interne, simple précision... superfétatoire ? On pourrait de plus le penser, à la lecture des travaux de chercheurs aussi différents que M. Wilmet et B. Comrie qui de façon similaire, font état de cette notion dans leur présentation générale de la catégorie de l'aspect, pour aussitôt l'abandonner lorsqu'ils passent à la description de l'aspect des différents temps. 
Dans les prolégomènes de la section sur l'aspect de sa Grammaire critique $\left(2010^{5}\right)$, M. Wilmet note :

Notre intention est d'explorer plus avant le «temps impliqué » en vue d'un inventaire aussi complet que possible des aspects, de leur combinatoire. (§ 171, p. 183)

Mais ensuite, lorsqu'il procède à ladite exploration, il ne fait plus état du temps impliqué et décrit les différents aspects comme différentes façons de représenter le procès « allant d'un terminus a quo ( $\alpha$ ) à un terminus ad quem $(\omega)$ » (p. 184).

B. Comrie pose une «difference between situation-internal time (aspect) and situation-external time (tense)» (1976:5), qui semble rencontrer la différence guillaumienne temps impliqué /temps expliquévii. Mais de fait, il use de la notion d'internal time, selon nous, de façon très lâche, seulement pour opposer le perfective comme "totality of the situation referred to without reference to its internal temporal constituency" (ibid:: 3 ), à l'imperfective comme "explicit reference to the internal temporal constituency of the situation; no reference to the beginning or to the end" (ibid:4).

On pourrait en conclure que, aussi pertinente soit-elle peut-être, la catégorie de temps interne n'est pas incontournable dans la description de l'aspect verbal, et qu'on peut aisément l'ignorer, en faire l'économie, ou la mentionner sans vraiment la faire travailler.

Il est pourtant au moins deux lieux du système où la notion de temps interne permet de fournir une explication consistante : le p. p. (§ 2.), et le passé composé (§3.).

\section{De l'aspect du p.p.}

Après avoir défini la valeur en langue du p.p. dans sa double dimension : temporelle et aspectuelle, nous expliciterons les faits de langue morphologiques et syntaxiques dont l'approche en termes de temps interme permet (seule ?) de rendre compte.

\subsection{Absence d'instruction temporelle}

Le p.p. est une des trois formes du mode atemporel et apersonnel « in posse » (Guillaume 1970 [1929] : 17), complémentairement à l'infinitif et au p. présent. Quels que soient leurs cadres théoriques, les linguistes s'accordent à dire que, comme les deux autres formes, le p.p. en français ne situe pas le procès dans le temps externe, d'où le manque de pertinence du terme de passé par lequel on l'oppose au participe présent (tout aussi mal nommé). Le p.p. ne donne pas d'instruction temporelle pour inscrire le procès dans l'une des trois époques : passée, présente ou future, ce qui se manifeste par le fait qu'il peut se combiner avec chacune d'elles pour construire les formes analytiques de l'aspect extensif : il avait/a/aura neigé.

\subsection{Instruction aspectuelle détensive}

L'infinitif représente le temps interne en seule tension (aspect tensif global : $\left(\frac{\text { tension }}{0}\right)$ ) ; le participe présent le représente dans son cours comme conversion de la tension en détension (aspect tensif cursif : $\left(\frac{\text { tension }}{\text { détension }}\right)$ ), le p.p. le représente d'une façon spécifique : au terme de son cours, en seule détension (aspect détensif $\left(\frac{0}{\text { détension }}\right)$ ).

Réécrivons notre analyse dans les termes de Reichenbach (1947) tels que retravaillés dans Azzopardi et Bres 2017. Nous reprenons le point S (speech point), considérons que R 
(reference point) peut être un point ou un intervalle, et reformulons le point $\mathrm{E}$ (event point) en intervalle du temps interne $E_{\mathrm{i}}-\mathrm{E}_{\mathrm{t}}$ du procès, ce qui permet de décrire le p.p. :

- temporellement (relation R/S) : le p.p. ne donne aucune instruction concernant la localisation du point de référence $\mathrm{R}$ par rapport au moment $\mathrm{S}$ de l'énonciation ;

- et aspectuellement (relation R/E) : le p.p. donne l'instruction de faire coïncider le point de référence $\mathrm{R}$ avec le terme $\mathrm{E}_{\mathrm{t}}$ du déroulement du temps interne du procès.

Compte tenu du non-marquage de la relation temporelle, nous pouvons décrire le p.p. comme une forme seulement aspectuelle, selon la formule : $\left[\mathrm{R}=\mathrm{E}_{\mathrm{t}}\right]$. Soit la figure 2 :

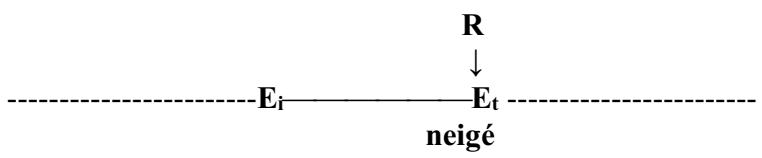

Figure 2. Aspect du p.p.

Par comparaison, le p. présent comme conversion de la tension du temps interne en détension relève de l'aspect cursif et aura pour formule : $\left[\mathrm{R} \subset \mathrm{E}_{\mathrm{i}}-\mathrm{E}_{\mathrm{t}}\right]$ (le point de référence $R$ se situe à l'intérieur de l'intervalle temporel $E_{\mathrm{i}}-\mathrm{E}_{\mathrm{t}}$ ), et pour figure :

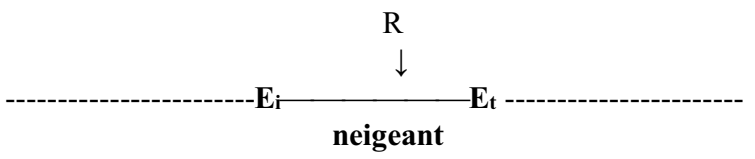

Figure 3. Aspect du p. présent

En tant que point ultime de la tension qui représente ce qui fait la spécificité du verbe, à savoir le temps interne, au terme du déroulement $E_{t}$ de la phase processuelle comme totalement dépensé, le p.p. est « la forme morte du verbe », (Guillaume 1971, Leçon du 24 février 1949: 170) : «ce qui subsiste du verbe après que la tension en est échappée » (Guillaume 1970 [1929] : 18).

C'est cette représentation aspectuelle détensive du temps interne qui fait la spécificité et l'unicité du p.p. : s'il est possible de rapprocher aspectuellement l'infinitif neiger du passé simple neigea sur la base de leur commune représentation globale du temps interne, et le p. présent neigeant de l'imparfait neigeait sur la base de leur commune représentation cursive du temps interne, aucune autre forme ne saurait être couplée avec le p.p. Son unicité tient à la façon unique dont il saisit le temps interne : au moment précis où il est représenté comme entièrement dépensé.

Cette singularité rend compte de façon satisfaisante de différents faits morphologiques et syntaxiques qui peuvent apparaître autrement mal expliqués ou inexpliqués.

\subsection{Faits morphologiques}

L'aspect détensif du p.p. permet de rendre compte du choix de cette forme dans la construction des formes analytiques ainsi que de sa morphologie singulière.

\subsubsection{P.p. et construction des formes analytiques}

Le français, comme les autres langues romanes, a construit son système des temps verbaux en doublant les formes synthétiques de tous les modes par des formes analytiques viii formées sur l'emploi du p.p. précédé d'un auxiliaire (être ou avoir) conjugué au temps de la forme simple correspondante, soit [auxiliaireêtre/avoir (conjugué) + p.p.], à la différence du latin, qui a construit l'essentiel de son système sur des formes synthétiques. 
Pourquoi les formes analytiques de la phase post-processuelle sont-elles construites sur le p.p. (il a neigé) et non sur l'infinitif (*il a neiger) ou sur le p. présent (*il a neigeant), alors que le prospectif (phase pré-processuelle) se construit sur l'infinitif (il va neiger), et que le p. présent a pu être utilisé pour construire la phase processuelle analytiquement, ce dont le tour il est mourant est la trace, tour que l'on trouve fort vivace notamment en anglais, en espagnol et en catalan (it is snowing, esta nevando, esta nevant : 'il neige') ${ }^{\mathrm{ix}}$ ?

La structure des formes post-processuelles en langue est la suivante : le procès atteint avec le p.p. le point ultime de sa tension, à savoir celui où le temps interne qui le définit est présenté comme écoulé ; il se voit renouvelé en tant que verbe par un auxiliaire qui en fait une nouvelle mise en tension :

Le système des aspects français repose entièrement sur une anastase - une résurrection du verbe - obtenue au moyen d'un auxiliaire. (...) Marché c'est le verbe qui n'a plus devant soi aucun devenir à dépenser, qui expire au terme de sa course. Le rôle de l'auxiliaire est de le ressusciter à ce moment d'expiration et d'en faire un nouveau verbe conjugué. (Guillaume 1992 : 24-25, Leçons de l'année 38-39)

Passons sur la métaphore vitaliste, voire christique ! C'est parce que le p.p. représente le temps interne détensivement qu'il entre dans la construction des temps analytiques, qui prennent en charge la phase post-processuelle, ce qui est interdit à l'infinitif et au p. présent dans la mesure où ils représentent le temps interne globalement ou dans son cours ${ }^{\mathrm{x}}$. L'auxiliaire vient au secours de la forme détensive du verbe, le p.p., pour lui permettre de redevenir " un verbe complet en deux mots » (Guillaume 1992:27), mais un verbe qui prend en charge la représentation non de la phase processuelle (tension), mais de la phase post-processuelle (extension) ${ }^{\mathrm{xi}}$.

Et complémentairement : si le p.p. n'entre pas dans la construction des formes des phases pré-processuelle et processuelle, c'est parce qu'il représente le temps interne au terme de son déroulement.

\subsubsection{Absence des formes analytiques et bi-analytiques du p.p.}

Si le p.p. entre dans la construction analytique et bi-analytique de toutes les autres formes verbales, dispose-t-il lui-même de telles formes ? Force est de constater que sur ce point il n'y a pas consensus entre grammaires de référence. Prenons un verbe intransitif comme arriver et un verbe transitif comme fermer: les formes étant arrivé et ayant fermé sont-elles les constructions analytiques des p.p. arrivé et fermé :

(a) arrivé, fermé > étant arrivé, ayant fermé

ou celles des p. présents arrivant ou fermant :

(b) arrivant, fermant > étant arrivé, ayant fermé ?

La plupart des grammaires (Grevisse et Goosse $\left(2008^{14}: \S 925\right)$, Chevalier et al. (1964 : 378), Arrivé, Gadet, Galmiche (1986 : 474), Riegel et al. ([1994] 2009 : 596)) optent pour la solution (a); d'autres, au contraire, (Wagner et Pinchon (1969), Wilmet $\left(2010^{5}: \S 211\right)$ ), pour la solution (b) ${ }^{\mathrm{xii}}$. La simple considération de la formation des formes analytiques à partir des formes synthétiques invite à invalider (a) et à accréditer (b) : étant arrivé, ayant fermé, du fait de l'auxiliaire au p. présent (étant, ayant), ne peuvent être que les formes analytiques du p. présent, en aucun cas les formes analytiques du p.p.

Mais alors quelle est la forme analytique du p.p. ? En vertu de la formation rappelée supra : [auxiliaire à la forme simple + p.p.], on devrait avoir :

(c) arrivé, fermé > été arrivé, eu fermé 
Ladite forme existe-elle? Si on trouve des énoncés à la forme synthétique du p.p. : Corinne arrivée, la porte fermée, il n'y a pas d'énoncés de forme analytique : *Corinne été arrivée, *la porte eu fermée, et donc il n'existe pas de forme analytique du p.p.

Cette conclusion suscite immédiatement l'objection suivante : les formes été arrivé ou eu fermé existent bel et bien dans les formes bi-analytiques, p. ex. pour l'infinitif : avoir été arrivé, avoir eu ferméxiii. Mais cette objection peut être levée aisément : ce sont là les formes infinitives surcomposées des infinitifs composés être arrivé, avoir fermé, formées régulièrement-:

(d) être arrivé, avoir fermé > avoir été arrivé, avoir eu fermé.

L'unité signifiante n'est pas avoir [été arrivé] ni avoir [eu fermé], qui réaliserait un p.p analytique; mais [avoir été] arrivé, [avoir eu] fermé, qui réalise un infinitif analytique $^{\mathrm{xiv}}$.

La conclusion s'impose que le p.p. n'existe qu'à la forme synthétique ${ }^{\mathrm{xv}}$. Ce fait prend toute sa signification de ce qu'il est la seule forme verbale ainsi défective : tous les autres temps de tous les autres modes disposent d'une forme synthétique, d'une forme analytique, et d'une forme bi-analytique, notamment les formes des autres modes non finis, le p. présent et l'infinitif :

\begin{tabular}{|c|c|c|c|}
\hline Aspect tensif & Aspect extensif & Aspect bi-extensif \\
\hline infinitif & arriver, fermer & $\begin{array}{c}\text { être arrivé, avoir } \\
\text { fermé }\end{array}$ & $\begin{array}{c}\text { avoir été arrivé, } \\
\text { avoir eu fermé }\end{array}$ \\
\hline p. présent & arrivant, fermant & $\begin{array}{c}\text { étant arrivé, ayant } \\
\text { fermé }\end{array}$ & $\begin{array}{c}\text { ayant été arrivé, } \\
\text { ayant eu fermé }\end{array}$ \\
\hline p. passé & arrivé, fermé & $\varnothing$ & $\varnothing$ \\
\hline
\end{tabular}

Tableau 4. Aspects des formes de l'in posse

Pourquoi, alors que tous les temps à tous les modes disposent d'une forme analytique, la seule forme où elle fait défaut est le p.p. ? L'aspect détensif $\left[\mathrm{R}=\mathrm{E}_{\mathrm{t}}\right]$ nous semble rendre compte de cette impossibilité : une forme «morte » ne peut être... doublement morte ! Ou, plus précisément : l'on ne saurait « ranimer» la forme «morte» du p.p. du verbe (arrivé, fermé) par une autre forme «morte », celle de l'auxiliaire (été, eu), ce qui rend compte de l'impossibilité de *été arrivé, *eu fermé. Corrobore ce fait la morphologie du p. présent: représentant le temps interne comme conversion de la tension en détension, le p. présent synthétique peut disposer d'une forme complémentaire analytique, composée seulement de détension : étant arrivé, ayant fermé. Le p.p., correspondant au point de détension du temps interne, ne saurait avoir une forme complémentaire analytique détensive : il est déjà luimême détensif.

Notre analyse rend compte de la parasynonymie entre la forme du p.p. (arrivé) et celle $\mathrm{du}$ p. présent analytique (étant arrivé), parasynonymie qui selon nous est à l'origine de l'analyse erronée de P. Imbs pour qui le p.p. aurait deux formes (cf. note 12) :

(2) Arrivés près de Bologne, nos amis se firent conduire à travers champs sur la route qui de Florence conduit à Bologne. (Stendhal, La Chartreuse de Parme, 1839) 
On peut gloser «arrivés près de Bologne » par «étant arrivés près de Bologne » : si le p.p. équivaut grosso modo à la forme analytique du p. présent, c'est que les deux peuvent prendre en charge la phase post-processuelle du procès.

\subsection{Faits syntaxiques}

La représentation détensive du temps interne par le p.p. explique trois faits syntaxiques : son accord en genre et en nombre avec le SN auquel il est incident, le fait qu'il ne réalise pas le pronom réfléchi des verbes pronominaux, et ses (im)possibilités en emploi nu.

\subsubsection{Accord en genre et en nombre}

La singularité du p.p. apparaît de façon particulièrement saillante lorsqu'on le rapproche, en emploi d'apposition, de la forme la plus voisine, le p. présent :

(3) elles (les femmes) n'ont que des apparences de foiblesse ; et fâchées que l'objet aimé exige d'elles autre chose qu'un coeur tendre, elles ne font que se prêter, pour ainsi dire, à ses propres foiblesses. (A.R. Lesage, Aventures du chevalier de Beauchêne, 1732)

(4) Mais déjà la Frimat et la Bécu, se fâchant, la maintenaient en place. (Zola, La Terre, 1887)

En (3), le p.p. fâchées prend la marque du féminin pluriel du pronom elles auquel il est incident syntaxiquement, alors que le p. présent se fâchant en (4), incident au SN féminin pluriel « la Frimat et la Bécu », ne prend pas la marque du genre et du nombre. Si le p.p. s'accorde comme un adjectif avec le SN auquel il est incident, c'est parce que, du fait de sa représentation détensive du temps interne, il se rapproche de l'adjectif et s'éloigne $\mathrm{du}$ verbe. Si les autres formes verbales, notamment une autre forme non finie comme le $\mathrm{p}$. présent, ne réalisent pas ce type d'accord, c'est parce que le temps interne qu'elles donnent à voir les prévient de glisser hors de la catégorie du verbe ${ }^{\mathrm{xvi}}$.

\subsubsection{Absence du pronom réfléchi}

Soit cette autre spécificité du p.p. en emploi nu : il ne réalise pas le pronom réfléchi du pronominal se fâcher (fâchées) en (3)), à la différence p. ex. du p. présent (se fâchant en (4)) (ou d'ailleurs de tout autre temps). Les rares linguistes qui, à notre connaissance, ont relevé ce fait, ne l'expliquent pas, ou pas vraiment. Ainsi Legendre (1989: 133) note : "the reflexive marker fails to appear because of the participial verbal morphology", mais n'explique pas en quoi la morphologie du participe rend compte de cette absence : vertu dormitive de l'opium ? L'aspect détensif du p.p. est là également un bon candidat : dans la construction pronominale, le pronom réfléchi sert à bloquer la tension du temps interne du procès sur le prime actant ${ }^{\text {xvii }}$, xviii; ce blocage n'ayant plus lieu d'être lorsque la forme verbale est détensive, son expression n'est alors plus nécessaire.

\subsection{3. (Im)possibilités du p.p. en emploi nu}

Le p.p. peut s'employer nu avec certains verbes, mais pas avec d'autres. Rappelons que par emploi $n u$ on vise les cas où le p.p. ne participe ni à la construction des formes analytiques (elle a aimé) ni à la construction passive (elle est aimée), à savoir lorsqu'il est incident à un actant sans la médiation de être ni de avoir, et fonctionne comme épithète, apposition, attribut de l'objet ou prédicat d'une participiale Soit les occurrences suivantes, représentatives des (im)possibilités : 
(2) Arrivés près de Bologne, nos amis se firent conduire à travers champs sur la route qui de Florence conduit à Bologne. (Stendhal, La Chartreuse de Parme, 1839)

(3) Elles (les femmes) n'ont que des apparences de foiblesse ; et fâchées que l'objet aimé exige d'elles autre chose qu'un coeur tendre, elles ne font que se prêter, pour ainsi dire, à ses propres foiblesses. (A.R. Lesage, Aventures du chevalier de Beauchêne, 1732)

(5) Ma porte fermée, quand l'obscurité eut envahi pour la première fois ma maison, une tristesse profonde s'étendit sur moi comme un suaire. (P. Loti, Aziyadé, 1879)

(6) Les israëlites allerent dans le désert de Sur, et (*marché) ayant marché dans cette solitude ils ne trouverent point d'eau. (Voltaire, $L a$ Bible enfin expliquée par plusieurs aumoniers, 1776)

(7) Et le prince, (*fermé) ayant fermé la porte, resta un moment dans le salon pour y lire la lettre que Murph venait de lui remettre. (E. Sue, Les Mystères de Paris, 1843)

Le p.p. peut être incident au prime actant des intransitifs qui construisent leur forme analytique sur l'auxiliaire être (intransitifs-être) ${ }^{\mathrm{xix}}$ comme arriver (2) (à l'exception de aller, cf. infra (8)), de certains pronominaux comme se fâcher (3), ainsi qu'au second actant des transitifs téliques (5) comme fermer. Mais il ne peut être incident au prime actant ni des intransitifs qui, (dans leur grande majorité), construisent leur forme analytique sur l'auxiliaire avoir (intransitifs-avoir) comme marcher (6) ni des transitifs (7) comme fermer $^{\mathrm{xx}}$ : dans ce cas, on fait usage de la forme analytique du p. présent (ayant $+\mathrm{V}_{\mathrm{p} . \mathrm{p} .}$ ).

Comment rendre compte de ce que le p.p. nu peut s'employer avec les intransitifs-être (2), avec certains pronominaux (3), ainsi qu'avec les transitifs en incidence au second actant (5) ; mais ne le peut ni avec les intransitifs-avoir (6) ${ }^{\mathrm{xxi}}$ ni avec les transitifs en incidence au prime actant (7) ? Remarquons que les verbes intransitifs du type de (2) ainsi que les verbes pronominaux (3) usent de être pour leurs formes analytiques (ils sont arrivés, elles se sont fâchées), forme qui sert également à la construction du passif périphrastique (5) : ma porte est fermée) ; alors que les autres intransitifs du type de (6) ainsi que les transitifs (à la voix active) (7) usent de avoir : ils ont marché, le prince a fermé la porte.

Qu'est-ce qui motive cette répartition? Et quelle corrélation entre l'emploi de être et la possibilité d'user du p.p. nu d'une part ; et l'emploi de avoir et son impossibilité d'autre part ? Nous avons montré (Bres et Le Bellec 2017) que les facteurs qui peuvent rendre compte de ces faits sont l'agentivité et la patientivité d'une part, et la transitionnalité d'autre part.

\subsubsection{Agentivité et patientivité}

Etre est un marqueur de la patientivité du sujet grammatical, avoir de son agentivité. Et ce qu'ont en commun les intransitifs-être, les pronominaux et la construction passive, c'est la dimension de patientivité du sujet ; comme ce qu'ont en commun les intransitifsavoir ainsi que la construction active, c'est la dimension d'agentivité du sujet.

La distinction des deux notions d'agentivité et de patientivité est particulièrement nette pour les verbes transitifs, comme fermer : la construction active ( $x$ fermer $y$ ) pose en sujet agentif $x$, là où la construction passive ( $y$ fermé (par $x$ )) pose $y$ en sujet patientif. La construction active en (7) - « le prince, *fermé (ayant fermé) la porte » n'admet pas le p.p. nu : l'acte de fermer, incident au sujet agentif le prince, demande le participe présent analytique ayant fermé. La construction passive en (5) - «Ma porte fermée, (...), une 
tristesse profonde s'étendit sur moi » admet le p.p. nu : l'acte de fermer est incident au sujet (ou contrôleur) patientif ma porte.

Les verbes intransitifs-être comme arriver, en vertu de leur appartenance d'origine à la voix moyenne en tant que déponents (Guillaume 1964 [1943]) posent leur prime actant à la fois comme agentif et comme patientif en ce qu'il est affecté par le procès ${ }^{x x i i}$ : l'emploi nu du p.p. est possible : (2) « Arrivés près de Bologne, nos amis (...) ». L'analyse vaut pour les verbes pronominaux qui, selon Guillaume, correspondent au développement d'une voix moyenne spécifique, dans laquelle « les fonctions d'agent et de patient se confondent en une seule personne » (Guillaume1974 : 174) : le pronominal se fâcher peut s'employer nu en incidence à ce prime actant agentif/patientif : (3) «(les femmes) n'ont que des apparences de foiblesse ; et fâchées que l'objet aimé exige d'elles $(\ldots) »$.

Les verbes intransitifs-avoir comme marcher ont un prime actant pleinement et seulement agentif qui n'admet pas le p.p. nu : l'acte de marcher, incident au sujet agentif les Israélites, demande le participe présent analytique ayant marché : (6) «les Israëlites (...), et ayant marché dans cette solitude (...) ».

Nous avons posé d'une part une corrélation entre la possibilité d'emploi nu du p.p. avec les intransitifs-être, certains pronominaux, ainsi qu'en incidence au second actant des verbes transitifs, et le fait que ces verbes (ou ces tours) impliquent une dimension de patientivité de l'actant auquel le p.p. est incident, ce que signalerait l'auxiliaire être; et d'autre part une corrélation entre l'impossibilité d'emploi nu du p.p. en incidence au prime actant des intransitifs-avoir et des transitifs, et le fait que leur actant auquel le p.p. est incident est seulement agentif, ce que signalerait l'auxiliaire avoir.

Mais sur quelle base se fait cette corrélation entre possibilité d'emploi nu du p.p. et patientivitéxxiii ; et impossibilité d'emploi nu du p.p. et agentivité ? Différons pour l'heure la réponse et présentons le second facteur, la transitionnalité.

\subsubsection{Transitionnalité}

Ce paramètre est défini par Martin (1988:4) comme «le fait que certains verbes aboutissent à un état résultant ». Ainsi en (2) («Arrivés près de Bologne, nos amis »), le procès arriver débouche sur un état résultant, que prend en charge le p.p. nu, qui peut être glosé à l'aide de être : nos amis sont arrivés, au sens de 'dans un état consécutif à l'acte d'arriver'.

La transitionnalité n'est le plus souvent pas prise en compte dans les analyses de l'aspect lexical ${ }^{\text {xxiv }}$, qui usent seulement de la quadripartition empruntée à Vendler (1967) pour opposer les états-activités comme atéliques aux accomplissementachèvement comme téliques (Garey 1957). Or la transitionnalité apparaît comme un critère à corréler avec l'emploi nu du p.p. Testons les différents types :

- intransitifs-être: des 28 verbes de cette catégorie, seul aller ne débouche pas sur un nouvel état du sujet consécutif à cet acte : c'est précisément celui qui n'accepte pas le p.p. en emploi nu:

(8) Et le roi (*allé) étant allé ce jour-là à Aigues-Mortes, il lui en parla durant son dîner. (R. Arnauld d'Andilly, Mémoires, 1667)

- pronominaux: si se fâcher se rencontre au p.p. nu (3) mais pas se rappeler, c'est que en (3) les femmes sont fâchées, alors que en (9), Albertine n'est pas *rappelée : 
(9) (*rappelé) s'étant rappelé qu'Elstir lui avait parlé de la duchesse comme de la femme de Paris qui s'habillait le mieux, le dédain républicain à l'égard d'une duchesse fit place chez mon amie à un vif intérêt pour une élégante. (M. Proust, À la recherche du temps perdu, 1922)

- transitifs : ces verbes, lorsqu'ils sont passivables, sont transitionnels. Si fermer, au p.p. nu, peut être incident au second actant (5) (la porte est fermée) mais pas au prime actant (7) (le prince *fermé la porte), c'est que l'état résultant du procès fermer affecte le second actant et non le prime actant.

- intransitifs-avoir: marcher ne débouche pas sur un état résultant, et ne dispose pas d'un emploi nu du p.p.

Il apparaît donc que la possibilité d'emploi nu du p.p. est liée à la conjonction de deux éléments : la patientivité de l'actant auquel il est incident, la transitionnalité du verbe. L'emploi nu est possible avec les procès transitionnels qui patientisent totalement ou partiellement l'actant auquel le p.p. est incident (intransitifs-être, certains pronominaux, transitif en incidence au second actant) ; et impossible avec les procès qui posent l'actant auquel il est incident comme seulement agentif (intransitif-avoir, transitif en incidence au prime actant) et /ou qui ne sont pas transitionnels.

Mais qu'est-ce qui explique que la possibilité d'emploi nu du p.p. soit liée à son incidence à un actant patientif (ou agentif-patientif), et à la transitionnalité du procès ; et son impossibilité à son incidence à un actant agentif, et / ou à la non-transitionnalité du procès? Notre hypothèse est que c'est là également l'aspect détensif du p.p. qui rend compte de ce fonctionnement.

C'est parce que le p.p. représente le temps interne au terme de son déroulement qu'il peut, en emploi nu, être incident à un actant patientif (ou agentif-patientif), mais pas à un actant agentif. Et c'est parce que le p.p. en emploi nu, grâce à sa représentation détensive du temps interne, peut signifier l'état résultant du procès affectant l'actant auquel il est incident qu'il demande un procès transitionnel.

Le lien entre emploi nu du p.p., représentation détensive du temps interne, patientivité (totale ou liée à l'agentivité) de l'actant, et transitionnalité du procès est confirmé par les (im)possibilités d'emploi nu du p. présent synthétique. Cette forme, qui représente le temps interne comme conversion de la tension en détension, soit donc d'aspect $\left[R \subset E_{i}-E_{t}\right]$, est possible en emploi nu :

(i) en incidence au prime actant agentif d'un intransitif-avoir comme marcher (10), ou d'un transitif comme fermer (11); d'un intransitif-être non transitionnel comme aller (12), et d'un pronominal non transitionnel comme se rappeler (13), tours dans lequels le p.p. est impossible :

(10) Louisa, marchant à côté de son fils, tâchait de l'abriter de la pluie.

(R. Rolland, Jean-Christophe, 1905)

(11) Puis, fermant la porte de l'appartement, il fit grincer la serrure. (E. Estaunié, L'Empreinte, 1896)

(12) Là-dessus, on se sépara, chacun allant à son service, (G. de Maupassant, Mademoiselle Fifi, 1881)

(13) Pendant ce temps, M. De Charlus, se rappelant qu'il était de race plus pure que la maison de France, se disait qu'il était bien bon de se faire tant de mauvais sang pour le fils d'un maître d'hôtel, (...). (M. Proust, À la recherche du temps perdu, 1922) 
(ii) en incidence à l'actant patientif d'un intransitif-être comme arriver, et d'un pronominal transitionnel comme se fâcher, tours dans lesquels le p.p. est également possible en emploi nu :

(14) Il eût craint (...) d'avoir l'air d'un provincial en extase devant les grâces d'une belle dame arrivant de la capitale. (Stendhal, La Chartreuse de Parme, 1839)

(4) Mais déjà la Frimat et la Bécu, se fâchant, la maintenaient en place. (Zola, La Terre, 1887)

Mais, et c'est là l'élément le plus intéressant pour nous, le p. présent n'est pas possible en emploi nu en incidence au second actant, à savoir l'actant uniquement patientif d'un verbe transitif comme fermer comme en (15) (cf. également (5) supra), à l'inverse du p.p. :

(15) Chez elle, la porte (*fermant, *ayant fermé) fermée, elle poussa son cri de détresse qui l'étouffait, et se laissa tomber dans un coin, la tête au mur. (P. Loti, Pêcheur d'Islande, 1886)

Soit donc le tableau suivant des (im)possibilités du p.p. et du p. présent en emploi nu :

\begin{tabular}{|l|c|c|}
\hline procès & p. présent & p.p. \\
\hline $\begin{array}{l}\text { intransitif-être } \\
\text { transitionnel }\end{array}$ & + & + \\
\hline $\begin{array}{l}\text { intransitif-être } \\
\text { non transitionnel }\end{array}$ & + & - \\
\hline intransitif-avoir & + & - \\
\hline $\begin{array}{l}\text { Pronominal } \\
\text { transitionnel }\end{array}$ & + & + \\
\hline $\begin{array}{l}\text { Pronominal non } \\
\text { transitionnel }\end{array}$ & + & - \\
\hline $\begin{array}{l}\text { transitif (prime } \\
\text { actant) }\end{array}$ & + & - \\
\hline $\begin{array}{l}\text { transitif (second } \\
\text { actant) }\end{array}$ & - & + \\
\hline
\end{tabular}

Tableau 5. (Im)possibilités des emplois nus des p. présent (synthétique) et passé selon le type de verbe

Notre explication $\mathrm{du}$ fonctionnement $\mathrm{du}$ p. présent en emploi nu nous semble confirmer celle fournie pour le p.p. : du fait de son aspect cursif $\left[R \subset E_{i}-E_{t}\right]$, le p. présent peut être employé nu avec tout type de verbe, sauf dans un seul cas : en incidence au second actant d'un verbe transitif. La raison est la suivante : le p. présent, représentant le temps interne comme conversion de la tension en détension, ne peut être incident à un actant seulement patientif, à savoir le second actant d'un transitif.

Soit donc : la représentation tensive du temps interne (p. présent) est compatible avec l'agentivité mais incompatible avec la patientivité ; la représentation détensive du temps interne (p.p.) est à l'inverse compatible avec la patientivité (ou une association de patientivité et d'agentivité), mais incompatible avec la seule agentivité. Nous retrouvons là, 
à propos de l'analyse du temps interne par les p. présent et passé, l'hypothèse que formulait R. Lafont il y a plus de 50 ans, à propos de la voix :

Il semble bien que la tension soit contradictoire du passif, et la détension de l'actif. Autrement dit, le passif coïncide avec la chute de tension, l'actif avec la progression tensive. (1967: 165)

Bilan : l'analyse aspectuelle du p.p. comme donnant à voir le temps interne au terme de son déroulement permet d'expliquer le fonctionnement syntaxique selon lequel, en emploi nu, il peut être incident à des actants patientifs (ou patientifs/agentifs), si le procès peut présenter un état résultant. Mais il ne peut être incident à des actants seulement agentifs, et/ou si le procès ne présente pas d'état résultant.

\section{Temps interne, fluence, ascendance / descendance}

Si nous avons montré en 2. l'importance du temps interne dans l'analyse morphosyntaxique du p.p. (et secondairement du p. présent), nous avons fait une appréhension statique de cette notion. Or le temps se caractérise, à l'inverse de l'espace, par sa fluence: le temps passe (ce qui soutendait notre analyse de la représentation du p. présent comme conversion de la tension du temps interne en détension). Cette dimension dynamique du temps est pertinente dans l'analyse du temps interne des procès selon les temps verbaux.

Revenons à $\mathrm{G}$. Guillaume qui propose d'appréhender la fluence du temps à partir des concepts de descendance et d'ascendance : la personne peut interpréter la fluence du temps selon l'orientation descendante, comme se déroulant du futur vers le passé (le temps est cette puissance qui emporte et détruit), ou selon l'orientation ascendante, comme se déroulant du passé vers le futur (le temps est le champ ouvert devant la personne pour qu'elle y inscrive son activité (Guillaume 1971 : 91-92), nous dirons sa praxis, entendue sous sa double forme de travail et de langage (Lafont 1978).

Nous avons eu l'occasion de développer l'hypothèse selon laquelle l'opposition imparfait / passé simple tenait à la différence de représentation du temps impliqué par le procès : descendante pour l'imparfait (conversion de la tension en détension en franchissement de la ligne du temps) / ascendante pour le passé simple (déroulement en seule tension du temps interne à partir de son incidence à la ligne du temps) (Bres 1997b, 1998).

Le sens de la fluence nous semble - également mais différemment - au principe de la distinction du passé simple (désormais PS) et du passé composé (désormais PC) en tant que temps narratifs.

Le PC a, au cours des siècles, suivi un chemin de grammaticalisation (commencé dès le bas-latin) en trois étapes selon Bybee et al. (1994: 105) : résultative $\rightarrow$ anterior/perfect $\rightarrow$ perfective/simple past. Cette troisième étape s'étale de l'ancien français au français moderne (cf. notamment Squartini et Bertinetto 2000, Caudal et Vetters 2007, Caudal 2015, Apothéloz 2016), avec une charnière entre français classique (XVII siècle) et français moderne (XVIII ${ }^{\mathrm{e}}$ siècle) (Caron et Liu 1999) : le PC, qui jusqu'alors n'admettait de circonstants qu'incluant $\mathrm{t}_{0}$, commence, à la fin du XVII ${ }^{\mathrm{e}}$ siècle, à pouvoir être flanqué de tout type de circonstant passé, construction qui se développe au XVIII ${ }^{\mathrm{e}}$ siècle. Le PC concurrence dès lors fortement le PS : il représente la phase processuelle globalement dans le passé, comme le PS : le PC de il a neigé en mars 2017 à Montpellier représente le temps interne du procès neiger dans son entièreté, comme le fait le PS : il neigea en mars 2017 à Montpellier. Mais cependant différemment.

Le PS représente le temps interne dynamiquement, depuis son début $\mathrm{E}_{\mathrm{i}}$ jusqu'à sa fin $\mathrm{E}_{\mathrm{t}}\left(\mathrm{R}_{\mathrm{i}} \rightarrow \mathrm{R}_{\mathrm{t}}\right)$ selon la fluence ascendante : 


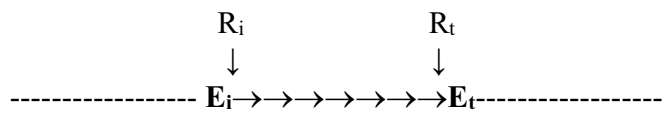

il neigea

Figure 4. Temps interne au PS

Le PC, de par le p.p. qui entre dans sa morphologie, saisit le temps interne sur sa borne terminale $E_{t}$, ce qui présuppose qu'il s'est déroulé entièrement. En fonction du cotexte, ce qui n'était que présupposé devient le posé : du fait du circonstant temporel dans il a neigé en mars 2017 à Montpellier, le PC donne à voir, à partir de la borne terminale, l'entier du temps interne du procès neiger dans sa phase processuelle, à l'époque passée, mais statiquement :

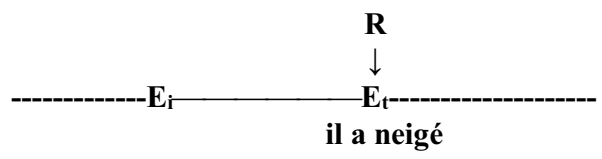

Figure 5. Temps interne au PC

Cette différence de représentation de la fluence du temps interne entre le PS et le PC est à l'origine de la différence de leur qualité narrative : excellente pour le PS, médiocre pour le PC. Et on peut faire l'hypothèse que l'usage quasi-systématique du PC en lieu du $\mathrm{PS}^{\mathrm{xxv}}$ dans l'écriture de L'Etranger d'A. Camus participe à la production du sentiment de l'absurde (Bres 2020).

La textualité narrative est habituellement définie comme enchaînement de propositions narratives (Labov 1978[1972]) dont l'ordre tend à (re)produire l'ordre des événements (du premier plan) (iconicité), ce qui implique que, d'une proposition à l'autre, le temps (raconté) auquel il est fait référence progresse. Nous avons proposé (Bres 1994) de parler pour cette structure de mise en ascendance. Soit la séquence de quatre propositions narratives empruntées à L'Etranger, au moment du meurtre :

(16) Tout mon être s'est tendu et j'ai crispé ma main sur le revolver. La gâchette a cédé, j'ai touché le ventre poli de la crosse.

D'un procès à l'autre, il y a progression ascendante externe :

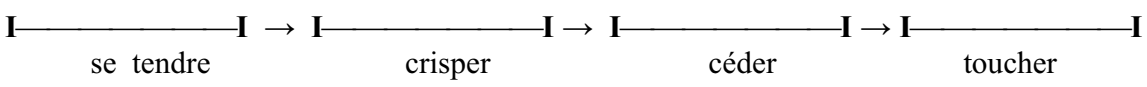

Figure 6. Ascendance externe

Lorsque les procès sont actualisés au PS, l'ascendance externe d'un procès au suivant est soutenue par l'ascendance du temps interne de chacun d'eux :

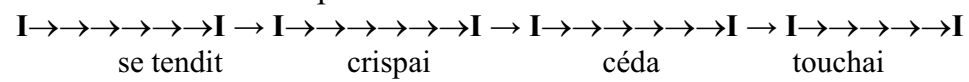

Figure 7. Ascendance interne des PS

Ascendance interne qui fait défaut lorsque les procès sont actualisés au PC :

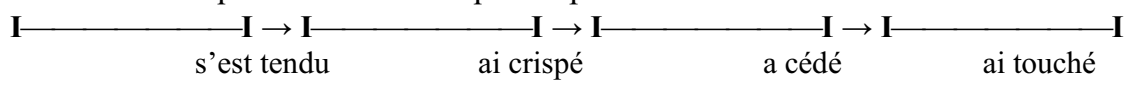

Figure 8. Défaut d'ascendance interne des PC 
Si le PS et le PC donnent à voir le temps interne globalement, ils le font différemment : le PS en le représentant dynamiquement, de $\mathrm{E}_{\mathrm{i}} \mathrm{à} \mathrm{E}_{\mathrm{t}}$, de façon ascendante, s'accorde avec la mise en ascendance narrative. Le PC, en le donnant à voir statiquement à partir de la borne terminale $\mathrm{E}_{\mathrm{t}}$, fait tendanciellement obstacle à ladite mise en ascendance narrative. C'est cette différence de saisie de déroulement du temps interne qui explique l'excellence du PS face à la médiocrité du PC dans l'actualisation d'une séquence de procès en relation de progression.

De nombreux auteurs ont noté, sans vraiment l'expliquer, qu'avec le passé composé se perdait la fluidité du récit, que chaque procès apparaissait non pas comme ouvert sur le suivant mais comme refermé sur lui-même :

Le passé composé, dit Marcel, c'est un temps imprécis, médiocre, bête et mou. « Nous avons été réveillés par la fusillade »... « Nous avons été »... Bon. Et alors? L'histoire est finie avant d'avoir commencé. Tandis que : « Nous fûmes réveillés par la fusillade »... Tu vois! Tu as dressé l'oreille. $\mathrm{Tu}$ attends la suite. (Audouard raconte Pagnol (Paris, Stock), apud Wilmet $1992: 24$ )

Dans une séquence de PS, chaque procès apparait comme conduisant au suivant. Dans une séquence de $\mathrm{PC}$, chaque procès apparait comme clos sur lui-même, détaché de la chaîne progressive qu'ils forment, ce qui participe activement à la production de l'impression selon laquelle,

une phrase de L'Étranger c'est une ile. Et nous cascadons de phrase en phrase, de néant en néant. C'est pour accentuer la solitude de chaque unité phrastique que M. Camus a choisi de faire son récit au parfait composé. (Sartre : Situations 1, 1947)

Est-ce parce que le PC n'est pas un bon outil pour raconter que le discours narratif journalistique, écrit comme oral, tend actuellement à développer le tour prospectif [aller au présent (et moins souvent à l'imparfait) + infinitif], comme dans l'occurrence infra (17)), retrouvant par là une forme que le français a connu jusqu'au début du XVII ${ }^{\mathrm{e}}$ siècle avant qu'elle ne devienne obsolète (Bres et Labeau 2013) ${ }^{\text {xxvi }}$ ?1993

(17) Dans le nord de la Birmanie, une longue histoire de tensions et de violences

La cause première [des tensions entre bouddhistes et musulmans] est à chercher du côté du colonisateur britannique, au temps de l'empire des Indes : en 1826, après s'être emparés de l'Arakan - l'actuel Etat Rakhine alors essentiellement bouddhiste -, les Anglais encouragent l'immigration de paysans musulmans de la partie orientale du Bengale voisin.

L'exploitation agricole de l'Arakan et l'essor du port d'Akyab, désormais appelé Sittwe, va ainsi susciter dans la région l'afflux de milliers d'agriculteurs bengalis, phénomène qui aura pour conséquence des bouleversements démographiques porteurs de tensions intercommunautaires ultérieures. A partir de la fin du XIX ${ }^{\mathrm{e}}$ siècle, le nombre de "Mahometans de Chittagong », comme l'on désignait à l'époque les ressortissants de cette ville située dans le sud de l'actuel Bangladesh, va progresser dans des proportions spectaculaires : entre 1890 et 1911, la population bengalie musulmane des districts d'Akyab ainsi que ceux de Maungdaw, Buthidaung et Rathedaung, épicentres des troubles actuels, va augmenter de $77 \%$.

La seconde guerre mondiale va aiguiser les tensions entre musulmans et bouddhistes. [...] (Le Monde, 13/09/2017) 
Là où le $\mathrm{PC}, \mathrm{du}$ fait du p.p., saisit rétrospectivement le temps interne à partir de la borne terminale, $v a+i n f$. le saisit prospectivement, s'accordant par là pleinement avec la marche en avant du récit, la mise en ascendance du temps : le sens de la narration est prospectif et non rétrospectif.

\section{Conclusion}

Nous sommes partis de la notion de temps impliqué par laquelle G. Guillaume définit l'aspect grammatical, et avons montré que cette notion, que nous avons renommée pour plus de lisibilité temps interne, permettait de décrire le système aspectuel des temps verbaux de l'indicatif en français. Nous avons développé ensuite l'hypothèse selon laquelle cette notion était plus heuristique encore dans l'explication de faits de langue et de discours peu, voire pas du tout, pris en compte par l'analyse linguistique : les particularités morphosyntaxiques du participe passé, l'emploi narratif du passé composé.

\section{Références bibliographiques}

Apothéloz, D., 2017, Le parfait d'expérience et l'évolution de la relation passé composé - passé simple. Dans : Sophie Prévost, Benjamin Fagard (éds), Le français en diachronie. Dépendances syntaxiques, morphosyntaxe verbale, grammaticalisation. Berne : Peter Lang, pp. 157-188.

Arrivé, M., Gadet, F., et Galmiche, M., 1986, La grammaire d'aujourd'hui, Paris : Flammarion.

Azzopardi S. et Bres J., 2017, Le système temporel et aspectuel des temps verbaux de l'indicatif (en français), Verbum, XXXIX, 1, 71-112.

Blanche-Benveniste C., 1998, L'usage prédicatif secondaire des participes passés. In Forsgren M, Jonasson K., \& Kronning H. (éd.). Prédication, assertion, information. Uppsala : Acta universitatis upsaliensis, 43-56.

Borel M., 2018, Formes surcomposées standard et formes surcomposées régionales, CMLF 2018.

Borel M., 2019, Les formes verbales surcomposées en français, thèse soutenue en juin 2019, à Fribourg.

Bres J., 1997a, Ascendance/descendance ; incidence/décadence : affaires de couples..., Cahiers de praxématique 29, 157-183.

Bres J., 1997b, Habiter le temps : le couple imparfait/passé simple en français, Langages 127, 77-95.

Bres J., 1998, Temps, praxis, sujet : de l'imparfait et du passé simple, L'information grammaticale 77, 33-37.

Bres J., 2015, De la défectivité de aller et de venir dans les périphrases d'ultériorité (il va pleuvoir) et d'antériorité (il vient de pleuvoir) proches à l'indicatif, L'Information grammaticale, 144, 27 33.

Bres J., 2020, Passé composé et insularisation phrastique dans L'Étranger d'A. Camus. De quelques problèmes de traduction en catalan et en castillan, in Eric Corre, Danh-Thành Do-Hurinville, Huy-Linh Dao (éd.), Linguistic approaches to Tense, Aspect, Modality, Evidentiality, based on the Novel L'Etranger ("The Stranger") by Albert Camus, and its Translations. Amsterdam: John Benjamins. Sous presse.

Bres J. et Labeau E., 2013, The narrative construction va + infinitive in Contemporary French: A linguistic phoenix rising from its medieval ashes?, Diachronica, 30, 3, 295-322.

Bres J. et Labeau E., 2015, Venir de (+ infinitive), An immediate anteriority marker in French, Diachronica 32:4, 530-570.

Bres J. et Labeau E., 2018, Des constructions en aller et venir grammaticalisés en auxiliaires, Syntaxe et sémantique 19, 49-86.

Bres J. et Le Bellec C., 2017, Du participe passé en français : fonctionnements, valeur en langue et effets de sens en discours, Linguisticae investigationes, $40: 2,274-303$.

Bres J. et Le Bellec C., 2019, Le participe passé et la construction analytique des temps verbaux en français, Journal of french language studies, 29, 323-348.

Bybee J., Perkins R. \& Pagliuca W. (1994). The evolution of grammar, Chicago: The University of Chicago Press. 
Caron P et Liu Y.-C., 1999, Nouvelles données sur la concurrence du passé simple et du passé composé dans la littérature épistolaire. L'Information grammaticale 82, 38-50.

Caudal P., 2015, Uses of the passé composé in Old French: evolution or revolution? Dans : Guéron J. (ed.), Sentence and Discourse, Oxford: Oxford University Press, 178-205.

Caudal P., Burnett H. \& Troberg M., 2016, Les facteurs de choix de l'auxiliaire en ancien français : étude quantitative. Dans: Prévost S. \& Fagard B. (éds), Le français en diachronie. Dépendances syntaxiques, Morphosyntaxe verbale, Grammaticalisation, Bern : Peter Lang, pp.237-265.

Caudal P., \& Vetters C., 2007, Passé composé et passé simple : Sémantique diachronique et formelle, Cahiers Chronos 16, 121-151.

Chevalier J.-Cl., Arrivé M., Blanche-Benveniste C. et Peytard J., 1964, Grammaire Larousse du français contemporain, Paris : Larousse.

Comrie B., 1976, Aspect. Cambridge: Cambridge University Press.

Creissels D., 2000, L'emploi résultatif de être + participe passé en français. Cahiers Chronos 6, 133142.

Creissels D., 2006, Syntaxe générale. Une introduction typologique 2. Paris : Lavoisier.

Damourette J. \& Pichon E., 1970 [1911-1936], Des mots à la pensée : essai de grammaire de la langue française. Tomes III et IV, Paris : d'Artrey.

Dik S., 1989, The Theory of Functional Grammar. Dordrecht: Foris.

Garey H. B., 1957, Verbal Aspect in French, Language 33, 91-110.

Gosselin L., 1996, Sémantique de la temporalité en français. Louvain-la Neuve : Duculot.

Gosselin L., 2011, L'aspect de phase en français : le rôle des périphrases verbales ", Journal of French Language Studies, 21/3, 149-171.

Gosselin L., 2017, Les temps verbaux du français : du système au modèle, Verbum XXXIV, 2017/1, 31-69.

Grevisse M. et Goosse A., [1936] 2008, Le Bon usage : grammaire française, Bruxelles : De Boeck, Duculot.

Grimshaw J., 1987 [1960], Unaccusative: An overview. NELS $17: 244-259$.

Guillaume G., 1964 [1933], Immanence et transcendance dans la catégorie du verbe. In Langage et science du langage. Paris : Nizet et Presses de l'université Laval, 46-58.

Guillaume G. (1964 [1943]). Existe-t-il un deponent en français? In Langage et science du langage. Paris : Nizet et Presses de l'université Laval, 127-142.

Guillaume G., 1970 [1919], Temps et verbe. Paris : Honoré Champion.

Guillaume, G., 1971, Leçons de linguistique 1948-1949, vol. 1, Québec, Les Presses de l'Université Laval et Paris, Klincksieck : Paris.

Guillaume G, 1992, Leçons de linguistique 1938-1939, vol. 12, Québec, Presses de l'Université Laval - Presses Universitaires de Lille.

Labov W., 1978, La transformation du vécu à travers la syntaxe narrative, dans Le parler ordinaire I, Paris, Minuit, 289-355.

Lafont R., 1967, La phrase occitane, Presses Universitaires de France.

Lafont R., 1978, Le travail et la langue, Paris : Flammarion.

Lagae V., 2005, Les formes en être + participe passé à valeur résultative dans le système verbal français. Cahiers Chronos 12, 125-142.

Legendre G. \& Sorace A., 2003, Auxiliaires et intransitivité en français et dans les langues romanes. In D. Godard (éd). Les langues romanes. Paris : CNRS Editions. 185-233.

Legendre G., 1989, Inaccusativity in French. Lingua 79, 95-164.

Martin R., 1988, Temporalité et classes de verbes. L'Information Grammaticale 39, 3-8.

Moignet G., 1981, Systématique de la langue française, Paris : Klincksieck.

Perlmutter, D. (1978). Impersonnal passives and the inaccusative hypothesis. Proceedings of the 4th Annual Meeting of the Berkeley Linguistics Society,157-190.

Reichenbach H., 1947, Elements of Symbolic Logic. New York : Macmillan \& Co.

Riegel M., Pellat J.-Cl. et Rioul M., 2009 [1994], Grammaire méthodique du français, Paris : Hachette.

Squartini Mario \& Bertinetto Pier Marco, 2000, The Simple and Compound Past in Romance languages, in Östen Dahl (ed.), Tense and Aspect in the Languages of Europe, Mouton-de Gruyter, 403-439.

Tesnière L., 1959, Éléments de syntaxe structurale. Paris : Klincksieck. 
Tournadre N., 2004, Typologie des aspects verbaux et intégration à une théorie du TAM. Bulletin de la Société de Linguistique de Paris, pp. 7-68.

Vendler Z., 1967, Verbs and times. The Philosophical Review 66 n², Cornell University Press, 143 160.

Wilmet M., 1992, Le passé composé: histoire d'une forme. Cahiers de praxématique 19, 12-36.

Wilmet M., 2010 , Grammaire critique du français. Paris : Hachette, Louvain-la Neuve : Duculot.

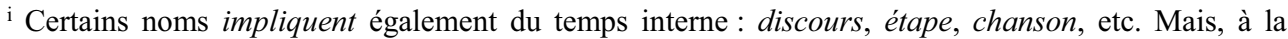
différence des verbes, ils ne le représentent pas en tension, et donc ne disposent pas de variation morphologique pour signifier différents aspects du déroulement de ce temps interne.

${ }^{i i} E_{i}$ et $E_{t}$ sont à distinguer des bornes initiale et terminale du procès lui-même, question qui relève de l'aspect lexical, et permet d'opposer les procès téliques (disposant d'une borne terminale (achèvement (exploser), accomplissement (traverser la rue)) aux procès atéliques (état (savoir), activité (courir)) (Garey 1957, Vendler 1967). Preuve de cette indépendance : le temps interne d'un procès atélique comme dormir peut être représenté jusqu'au terme de son déroulement $\left(\mathrm{E}_{\mathrm{t}}\right)$ par le passé simple (Corinne dormit toute la nuit); le temps interne d'un procès télique comme sortir peut être représenté dans le cours de son déroulement, donc avant la borne terminale intrinsèque du procès, par l'imparfait (Corinne sortait lorsque...).

iii Défectif, car il ne se réalise qu'au présent et à l'imparfait (Bres 2015).

${ }^{\text {iv }}$ Egalement défectif: on le trouve au présent, au futur et au conditionnel, à l'imparfait, mais pas au passé simple.

${ }^{v}$ M. Borel, dans sa thèse Les formes verbales surcomposées en français, soutenue en juin 2019, fournit quelques occurrences de ces formes bi-extensives prospectives qui sont très peu usitées. En revanche, elle n'a pas relevé d'occurrences (ni de mention dans les grammaires) de formes biextensives rétrospectives.

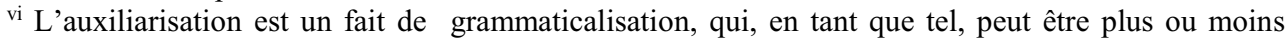
avancé : l'auxiliarisation est très avancée avec être et avoir; l'est moins pour aller, venir (de), être en train de; elle est encore moins avancée pour commencer, finir, etc. (Gosselin 2011, voir notamment la distinction entre auxiliaires de visée aspectuelle et coverbes de phase). Nous ne prendrons pas en compte ici ces dernières possibilités.

${ }_{\text {vii }}$ B. Comrie ne mentionne pas les travaux de G. Guillaume. Lors d'une conversation que nous avons eue avec lui, il nous a dit s'en être inspiré pour sa distinction internal time / external time.

viii Et en redoublement pour les formes bi-analytiques (surcomposées).

ix Notons également que la phase processuelle a pu être construite dans l'ancienne langue sur le $\mathrm{p}$. présent précédé de l'auxiliaire aller (il va neigeant), tour très vivant encore de nos jours en espagnol et en catalan : va nevando, va nevant : 'il neige'.

${ }^{x}$ Possibilité qui se réalise notamment avec l'auxiliaire aller: Corinne va chanter prend en charge la phase pré-processuelle; Corinne va chantant, la phase processuelle.

${ }^{x i}$ Notons que sporadiquement et brièvement (fin $\mathrm{XVI}^{\mathrm{e}}$-début $\mathrm{XVII}{ }^{\mathrm{e}}$ siècle) le p.p. a pu être utilisé dans la construction d'un tour pré-processuel :

(1) Monsieur, de Viole s'en va mort si vous ne luy aydez, car sa sentence est arrestée, et ceste nuict le doivent estrangler en prison [...], (B de Montluc, Commentaires, 1562) (Bres et Luxardo 2020).

xii Imbs (1960 : 161) propose une autre solution : le p.p. aurait « deux formes : ou bien il s'emploie avec les participes auxiliaires ayant ou étant, ou bien il s'emploie seul », sans expliquer les raisons de cette (bizarre) dualité, puisque seul le p.p. en disposerait.

xiii Moignet (1981: 60) : « [la forme du participe passé eu déjeuné] est, dans l'aspect transcendant, la forme conclusive permettant de construire l'aspect bi-transcendant». Wilmet $\left(2010^{5}: \S 211\right)$ partage cette analyse.

xiv Nous ne prétendons pas régler ainsi la question complexe de la formation des formes bianalytiques, notamment le fait qu'un verbe comme sortir dispose de deux formes de passé surcomposé : j'ai été sorti, à valeur résultative; et je suis eu sorti, à valeur expérientielle. Voir Borel 2018.

${ }^{x v}$ Blanche-Benveniste (1998:54) parvenait à semblable conclusion. 
xvi Là où le p.présent représente le temps interne dans son cours (Corinne, convainquant son ami (...)), l'adjectif verbal correspondant signifie un état ou une propriété et s'accorde (Corinne est très convaincante).

xvii Nous utilisons la terminologie issue de l'analyse de la valence verbale par Tesnière (1959: 239).

xviii Dans un autre cadre théorique, mais dans le même ordre d'idée, D. Creissels analyse « se comme un opérateur sur la valence » $(2006: 28)$.

xix Nous ne reprenons pas les termes d'inaccusatif $(\approx$ intransitif-être $)$ et d'inergatif $(\approx$ intransitifavoir) proposés, pour l'analyse des verbes intransitifs, par l'hypothèse inaccusative (Perlmutter 1978, Grimshaw 1987, Legendre 1989, Legendre \& Sorace 2003). Ces travaux font l'hypothèse qu'en structure profonde l'argument des inergatifs correspond à un sujet, alors que celui des inaccusatifs correspond à un objet. Les inaccusatifs ont pour sujet un argument interne non agentif mais affecté par le procès, et sélectionnent préférentiellement l'auxiliaire être (arriver, venir, sortir, mourir, tomber, etc.). Les inergatifs ont pour sujet un argument externe agentif, et sélectionnent préférentiellement l'auxiliaire avoir (courir, dormir, travailler, etc.). Nous ne partageons pas cette hypothèse selon laquelle le sujet d'un inaccusatif en structure de surface est en structure profonde un objet direct; mais nous nous accordons avec elle sur le fait que le sujet des verbes inaccusatifs (intransitifs-être), à la différence du sujet des verbes inergatifs (intransitifs-avoir), est affecté par le procès et a une composante de patientivité. Ceci le rapproche, par certains comportements syntaxiques comme précisément l'emploi nu du p.p., de l'objet direct des verbes transitifs.

${ }^{x x}$ Notre description confirme Legendre et Sorace (2003) et Helland (2014).

${ }^{x x i}$ A quelques exceptions près, explicitées dans Bres et Le Bellec 2017.

xxii «Dans j'ai fini, ce n'est pas moi sujet, qui suis fini; c'est la chose que j'avais entreprise. Le rapport considéré est celui du sujet à l'objet. Dans je suis sorti, c'est au contraire moi, sujet, qui suis sorti. Le rapport en cause est celui du sujet au verbe ». (Guillaume 1964 [1943] : 132)

xxiii Va dans le sens de notre analyse le fait que Damourette et Pichon (1970 [1911-1936]) nomment le p.p. « patiental» (III, § $863 ; \mathrm{IV}, \S 1171)$.

xxiv Voir toutefois, sous le nom de résultativité, pour la description de la valeur de certains tours composés de être + p.p. comme Corinne est sortie depuis 10', Bybee et al. 1994, Creissels 2000, Lagae 2005.

${ }^{x x v}$ On relève seulement 7 occurrences de PS : «Inadvertances » (Wilmet $1992: 28$ )?

xxvi Forme qui a réussi en catalan (et dans certains dialectes occitans), où elle est devenue la forme de base de la narration rétrospective: « va entrar al camerino en silenci, va seure i va esperar » (J. Cabré, Viatge d'hivern) (littéralement : 'il va rentrer dans la cabine, il va s'asseoir et va attendre'). 